\title{
Acute Coronary Syndrome in Idiopathic Inflammatory Myopathies: A Population-based Study
}

\author{
Valérie Leclair, John Svensson, Ingrid E. Lundberg, and Marie Holmqvist
}

\begin{abstract}
Objective. Evidence suggests an increased risk of cardiovascular (CV) diseases, including acute coronary syndrome (ACS), in idiopathic inflammatory myopathies (IIM). The aim of this study was to investigate the risk of ACS in an incident IIM cohort compared to the general Swedish population. Methods. A cohort of 655 individuals with incident IIM and 6813 general population comparators were identified from national registries. IIM subjects were diagnosed from 2002 to 2011. Followup started at IIM diagnosis and corresponding date in the general population. ACS, CV comorbidities, and $\mathrm{CV}$ risk factors were defined using International Classification of Diseases codes. Incidence rates including 95\% CI were calculated. Cox proportional hazards models were used to compare the risk of ACS in patients with IIM and the general population. The competing risk of death was accounted for using competing risk regression models.

Results. The incidence rate of ACS in IIM was higher than in the general population, particularly within the first year of diagnosis and in older individuals. The overall ACS incidence rate in IIM was 15.6 (95\% CI 11.7-20.4) per 1000 person-years, with an HR of 2.4 (95\% CI 1.8-3.2) compared with the general population. When accounting for the competing risk of death, the risk of ACS in IIM remained increased with a cumulative incidence of $7 \%$ at 5 years compared to $3.3 \%$ in the general population.
\end{abstract}

Conclusion. IIM individuals are at higher risk of ACS, particularly within the first year after diagnosis. (First Release August 15 2019; J Rheumatol 2019;46:1509-14; doi:10.3899/jrheum.181248)

Key Indexing Terms: DERMATOMYOSITIS
POLYMYOSITIS

\section{ACUTE CORONARY SYNDROME}

Idiopathic inflammatory myopathies (IIM) are a group of chronic autoimmune multisystemic diseases often characterized by muscle inflammation and muscle weakness. The major IIM subsets are dermatomyositis (DM), polymyositis (PM), inclusion body myositis (IBM), and immune-mediated necrotizing myopathies ${ }^{1}$. Morbidity and mortality are still higher in IIM than in the general population despite advances

From the Unit of Clinical Epidemiology, and the Division of Rheumatology, Department of Medicine, Solna, Karolinska University Hospital, Karolinska Institutet, Stockholm, Sweden; Division of Rheumatology, Jewish General Hospital, Montreal, Quebec, Canada.

Dr. Lundberg was supported by grants from the Swedish Research Council, the Swedish Rheumatism Association, King Gustaf V 80 Year Foundation, and the Stockholm County Council (ALF project). Dr. Holmqvist was supported by grants from the Swedish Rheumatism Association, the King Gustaf V 60 Year Foundation, the Nanna Svartz Foundation, and the Stockholm County Council (ALF project).

V. Leclair, MD, Unit of Clinical Epidemiology, Department of Medicine, Solna, Karolinska University Hospital, Karolinska Institutet, and Division of Rheumatology, Jewish General Hospital; J. Svensson, PhD, Unit of Clinical Epidemiology, Department of Medicine, Solna, Karolinska University Hospital, Karolinska Institutet; I.E. Lundberg, $M D, P h D$, Division of Rheumatology, Department of Medicine, Solna, Karolinska University Hospital, Karolinska Institutet; M. Holmqvist, MD, PhD, Unit of Clinical Epidemiology, and Division of Rheumatology, Department of Medicine, Solna, Karolinska University Hospital, Karolinska Institutet.

Address correspondence to Dr. V. Leclair, Karolinska Institutet, Unit of Clinical Epidemiology, Karolinska Hospital T2, SE-17176, Stockholm, Sweden.E-mail: valerie.leclair@ki.se

Accepted for publication February 26, 2019. in the field and introduction of novel therapies ${ }^{2,3}$. Evidence suggests an increased risk of cardiovascular (CV) disease, such as arrhythmias, congestive heart failure, and acute coronary syndrome (ACS) in IIM individuals ${ }^{4,5,6,7,8,9}$. The burden of $\mathrm{CV}$ diseases is of major concern in several rheumatologic conditions, notably rheumatoid arthritis and systemic lupus erythematosus ${ }^{10,11}$. In IIM, the higher prevalence of traditional CV risk factors coupled with a chronic inflammatory state and possibly vasculitis could account for an added ACS risk ${ }^{12,13,14,15}$. A majority of published studies to date have investigated the risk of ACS in prevalent IIM cohorts that lacked comparators and/or used combined CV endpoints ${ }^{4,5,6,7}$. A previous Canadian population-based study reported a 3- to 4-fold increased risk of myocardial infarction in individuals with DM and PM after adjustment for medication use ${ }^{9}$. However, unstable angina was not considered, stratification for sex or age was not included, and the effect of time on myocardial infarction incidence was assessed only up to 5 years after diagnosis. The aim of the present study was to investigate the risk of ACS in a large incident IIM cohort compared to the general Swedish population.

\section{MATERIALS AND METHODS}

Study design. This population-based cohort study includes patients with incident IIM (exposed) and matched comparators from the general population (unexposed).

Personal non-commercial use only. The Journal of Rheumatology Copyright @ 2019 . All rights reserved. 
Setting. The Swedish healthcare coverage is universal; thus all residents have access to publicly funded inpatient and outpatient healthcare. IIM individuals are usually diagnosed and followed by internists, rheumatologists, dermatologists, or neurologists practicing in hospital-based settings. The unique personal identification number given to all residents enables data retrieval from national administrative databases and clinical registries, with linkage of demographic, morbidity, and mortality data.

Study population. The Swedish National Patient Register (NPR; 1964-2012) indexes data on hospitalizations from 1987 and outpatient visits from 2001 Clinicians are allowed to allocate a main diagnosis and up to 10 contributory diagnoses for each hospitalization. The NPR coverage is almost universal for hospitalizations, and most patients with IIM (95\%) are followed by hospital-based specialists, resulting in a high coverage of outpatient visits in that population. The adult ( $\geq 18 \mathrm{yrs}$ ) incident IIM cohort was identified from the NPR using the International Classification of Diseases, 10th revision (ICD-10) codes for IIM (juvenile DM M33.0, DM M33.1, PM M33.2, 33.9, and IBM G72.4). The IIM case definition used in this study was used in previous publications and showed a positive predictive value (PPV) and a sensitivity of $>85 \%$ for all ICD codes except IBM ${ }^{16,17}$. Based on this algorithm, to be included in our cohort an individual was required to have (1) $\geq 2$ visits indexed with an IIM diagnosis by a specialist (dermatologist, internist, neurologist, or rheumatologist); (2) an initial visit between 2002 and 2011; and (3) a followup visit within 1 year listing an IIM code. We had access to data from 2001 to 2012, allowing us to exclude prevalent IIM cases that had received an IIM code in the year prior to the study period (2001) while following up on cases included in 2011. This approach accurately identified individuals with DM, but PM and IBM are considered together under "other IIM" because of coding overlap. Each patient with IIM was matched up to 1:10 on age, sex, and place of residence with randomly selected individuals from the general population identified through the Swedish Population Register. Comparators were required to be alive and residing in Sweden at the index date of their matched patient with IIM. This study received approval from the Regional Ethical Committee of Stockholm (2017/2000-31).

Definition of outcome. The primary outcome was the occurrence of the first-ever ACS during followup period. ICD-10 codes used to identify ACS from hospitalization records as primary discharge diagnosis and cause of death register were I20.0 for unstable angina and I21 (all subcategories) for acute myocardial infarction including non-ST-elevation (NSTEMI) and ST-elevation myocardial infarction (STEMI; Supplementary Table 1, available with the online version of this article). ACS identification using ICD codes has been shown to be valid, and the definition of ACS used in this study previously showed a PPV of $95 \%^{18,19}$. All individuals with a history of ACS at baseline were excluded.

Covariates. Sex and date of birth were extracted from the Swedish Population Register. Presence of CV comorbidities and risk factors at baseline were identified using in- and outpatient visits from the NPR indicating either stroke (I61, I63), atrial fibrillation (I48), stable angina (I20.9), heart failure (I42, I50), diabetes (E10, E11), and/or hypertension (I10, I11, I12, I13, I15) in the year preceding the index date $20,21,22,23,24$. In addition, the Prescribed Drug Register (PDR), including data on prescriptions filled across Sweden since 2006, was used to identify individuals treated for $\mathrm{CV}$ diseases or risk factors, and those exposed to medications that increase $\mathrm{CV}$ risk in the 6 months preceding the index date. The PDR was also used to determine immunosuppressive therapies received \pm 2 months from index date (Supplementary Table 2, available with the online version of this article). Educational level was retrieved from the Longitudinal Integration Database for Health Insurance and Labour Market Studies and was used as a measure of socioeconomic status ${ }^{25}$.

Index date and followup. The date of the second visit recorded was considered the index date in the IIM cohort. The followup started at this point and ended at first ACS, first emigration, death, or December 31, 2013, whichever occurred first.

Statistical analyses. Descriptive statistics were used to summarize charac- teristics of individuals with IIM and general population comparators at baseline and at first ACS. Crude incidence rates per 1000 person-years (PY) were calculated by dividing the number of ACS events by the followup period and were stratified by sex, age at diagnosis, and IIM subsets. CI were calculated assuming a Poisson distribution using the exact method ${ }^{26}$. The association between IIM and ACS was estimated using Cox proportional hazards models and expressed as HR. This approach considers that ACS and death are independent, while they are in fact competing. To account for the competing risk of death, the subdistribution HR (sd-HR) and cumulative incidences of ACS at 1,5, and 10 years for both groups were estimated using Fine and Gray competing risk regression models ${ }^{27}$. All models were adjusted for age at index date as a continuous variable, birth year, year of IIM diagnosis, sex, and residential area. To determine whether the risk of ACS was higher at diagnosis, HR of ACS stratified by time since index date $(<1$, $1-5,5-12$ yrs) were obtained using time-dependent covariates. A $p$ value of $<0.05$ was considered statistically significant. All analyses were performed using SAS software package version 9.4 (SAS Institute), R version 3.3.3 (R Foundation for Statistical Computing), and STATA IC 11.2 (StataCorp).

\section{RESULTS}

We included 655 incident IIM individuals and 6813 age- and sex-matched general population comparators. Sixty-one patients with IIM and 287 comparators were excluded given prior ACS. Baseline characteristics of both groups are presented in Table 1. Fifty-six percent were women and one-third of the patients had DM. The prevalence of atrial fibrillation and hypertension was slightly lower in the general population comparators than in patients with IIM. The subgroup of individuals with available medication data revealed a higher proportion of IIM subjects exposed to CV

Table 1. Baseline characteristics of incident IIM cases and matched general population comparators.

\begin{tabular}{|c|c|c|}
\hline Characteristics & $\begin{array}{l}\text { Patients with IIM, } \\
\qquad \mathrm{n}=655\end{array}$ & $\begin{array}{c}\text { General Population, } \\
n=6813\end{array}$ \\
\hline Followup, yrs, median (IQR) & $4.5(2.5-8.0)$ & $6.0(3.4-8.9)$ \\
\hline Women & $367(56)$ & $3821(56)$ \\
\hline Age, yrs, mean \pm SD & $60 \pm 15$ & $61 \pm 15$ \\
\hline \multicolumn{3}{|l|}{ Age group, yrs } \\
\hline$<56$ & $223(34)$ & $2242(33)$ \\
\hline $56-67$ & $215(33)$ & $2255(33)$ \\
\hline $68-90$ & $217(33)$ & $2316(34)$ \\
\hline \multicolumn{3}{|l|}{ Educational level, yrs } \\
\hline$<10$ & $205(31)$ & $2172(32)$ \\
\hline $10-12$ & $269(41)$ & $2741(40)$ \\
\hline$>12$ & $171(26)$ & $1795(26)$ \\
\hline Missing & $10(2)$ & $105(2)$ \\
\hline \multicolumn{3}{|l|}{ Diagnosis } \\
\hline Dermatomyositis & $218(33)$ & \\
\hline Other IIM & $437(67)$ & \\
\hline \multicolumn{3}{|l|}{ Cardiac comorbidities } \\
\hline Stroke & $15(2)$ & $166(2)$ \\
\hline Atrial fibrillation & $40(6)$ & $256(4)$ \\
\hline Stable angina & $21(3)$ & $167(2)$ \\
\hline Heart failure & $20(3)$ & $103(2)$ \\
\hline Diabetes & $28(4)$ & $253(4)$ \\
\hline Hypertension & $88(13)$ & $591(9)$ \\
\hline
\end{tabular}

Values are n (\%) unless otherwise specified. IIM: idiopathic inflammatory myopathy; IQR: interquartile range. 
drugs than the comparators (Table 2). The use of statins or fibrates was however lower in the patients with IIM, but a higher proportion of them were exposed to acetylsalicylic acid. As expected, a large proportion of the IIM group received immunosuppressive drugs around the index date. Fifty-three ACS occurred in the IIM group compared to 313 in the general population with a similar age and sex distribution (Table 3). Patients with IIM experienced their first ACS sooner in the followup period than the comparators [median (interquartile range) 2.4 (1.0-4.6) vs 3.5 (1.8-6.0) yrs)].

Incidence rate of ACS. The crude incidence rate for ACS in the IIM cohort was 15.6 (95\% CI 11.7-20.4) per 1000 PY, and 7.5 (95\% CI 6.7-8.4) per $1000 \mathrm{PY}$ in the general population (Table 4). The age- and sex-adjusted rate difference was 5.7 (95\% CI 2.2-9.3). The incidence rates of NSTEMI and STEMI were higher in the IIM group. In the general population, ACS incidence rates were higher in males, and in older individuals. In the IIM group, older subjects and those with inflammatory myopathies other than DM had the highest incidence rates. In contrast with the general population, incidence rates in women and men were similar in the exposed group.

Association between IIM and ACS. The overall age-, sex-,

Table 2. Baseline characteristics of incident IIM cases and matched general population comparators with available medication records.

\begin{tabular}{lcc}
\hline Characteristics & $\begin{array}{c}\text { Patients with IIM, } \\
\mathrm{n}=397\end{array}$ & $\begin{array}{c}\text { General Population, } \\
\mathrm{n}=4166\end{array}$ \\
\hline Followup, yrs, median (IQR) & $\begin{array}{c}3.5(2.3-5.5) \\
\text { Women }\end{array}$ & $\begin{array}{c}4.1(2.8-6.0) \\
2273(55)\end{array}$ \\
Age, yrs, mean \pm SD & $61 \pm 15$ & $62 \pm 15$ \\
Diagnosis & & \\
Dermatomyositis & $144(36)$ & \\
Other IIM & $253(64)$ & \\
Cardiac comorbidities & & $116(3)$ \\
Stroke & $11(3)$ & $193(5)$ \\
Atrial fibrillation & $27(7)$ & $119(3)$ \\
Angina & $15(4)$ & $85(2)$ \\
Heart failure & $14(4)$ & $175(4)$ \\
Diabetes & $19(5)$ & $470(11)$ \\
Hypertension & $76(19)$ & $1589(38)$ \\
Medications & & $514(12)$ \\
Cardiovascular drugs & $231(58)$ & $261(6)$ \\
$\quad$ Acetylsalicylic acid & $79(20)$ & $664(16)$ \\
Antidiabetics & $43(11)$ & $510(12)$ \\
Statins, fibrates & $46(12)$ & $44(1)$ \\
NSAID, COX-2 inhibitors & $101(25)$ & $148(4)$ \\
Hormone therapy & $5(1)$ & $42(1)$ \\
Corticosteroids & $289(73)$ & \\
Other immunosuppressors* & $178(45)$ & \\
\hline
\end{tabular}

Values are $\mathrm{n}(\%)$ unless otherwise specified. * Including calcineurin inhibitors, methotrexate, azathioprine, mycophenolate mofetil, and hydroxychloroquine. IQR: interquartile range; IIM: idiopathic inflammatory myopathy; NSAID: nonsteroidal antiinflammatory drugs; COX: cyclooxygenase.
Table 3. Characteristics of study population at first acute coronary syndrome.

\begin{tabular}{lcc}
\hline Characteristics & Patients with IIM & General Population \\
\hline No. events & 53 & 313 \\
Unstable angina & $2(4)$ & $28(9)$ \\
NSTEMI & $17(32)$ & $103(33)$ \\
STEMI & $34(64)$ & $182(58)$ \\
Women & $30(57)$ & $136(43)$ \\
Age at event, yrs, mean (SD) & $70(11)$ & $70(11)$ \\
Time to event, yrs, median (IQR) & $2.4(1.0-4.6)$ & $3.5(1.8-6.0)$ \\
Cardiac comorbidities & & \\
Stroke & $1(2)$ & $16(5)$ \\
Atrial fibrillation & $6(11)$ & $19(6)$ \\
Stable angina & $4(8)$ & $28(9)$ \\
Diabetes & $4(8)$ & $30(10)$ \\
Hypertension & $10(19)$ & $46(15)$ \\
Heart failure & $2(4)$ & $10(3)$ \\
\end{tabular}

Values are $\mathrm{n}(\%)$, unless specified. IIM: idiopathic inflammatory myopathy; STEMI: ST-elevation myocardial infarction; NSTEMI: non-STEMI; IQR: interquartile range.

and residential area-adjusted HR comparing the IIM cohort and the general population was 2.4 (95\% CI 1.8-3.2). The increased relative risk was highest in women, in the 68- to 90-year-old subgroup and in the "other IIM" group compared with the general population (Table 4). The relative risk of ACS in patients with DM was similar to the general population. When stratified by time since diagnosis, the HR for ACS was highest within a year from IIM diagnosis (HR 3.6, 95\% CI 1.9-6.7) and decreasing thereafter (Table 5). When the association of IIM and ACS was estimated by a competing risk model, considering death the competing event, the overall sd-HR was 1.9 (95\% CI 1.4-2.5). The cumulative incidence of ACS estimated using Fine and Gray competing risk models adjusted for age, sex, and place of residence at 1,5 , and 10 years was $2 \%$ (95\% CI 1.2-3.4), $7.0 \%$ (95\% CI 5.2-9.5), and $12.0 \%$ (95\% CI 8.9-16) in the IIM cohort, and $0.62 \%(0.46-0.83), 3.3 \%(2.9-3.8)$ and $6.9 \%$ (6.1-7.8) in the general population, respectively.

\section{DISCUSSION}

In this large population-based nationwide study looking at ACS risk in IIM, we found an increased incidence and relative risk of ACS in IIM individuals compared with the general population. In our IIM cohort, the ACS incidence rate was 15.6 (95\% CI 11.7-20.4) per $1000 \mathrm{PY}$ and the HR 2.4 (95\% CI 1.8-3.2) for IIM compared to general population comparators. Because patients with IIM have a significantly higher early mortality rate compared with the general population, it is important to take death into account when interpreting their risk of experiencing an $\mathrm{ACS}^{3}$. When accounting for the competing risk of early death, the sd-HR of ACS in our IIM cohort remained increased at 1.9 (95\% CI 1.4-2.5), with a cumulative incidence of ACS of $7 \%$ at 5 years compared to $3.3 \%$ in the general population. In a

Personal non-commercial use only. The Journal of Rheumatology Copyright @ 2019 . All rights reserved. 
Table 4. Number of ACS, followup, incidence rates, rate differences, and HR for ACS overall and stratified by sex, age group, and IIM subset for all the patients with IIM and matched general population comparators included in the study.

\begin{tabular}{|c|c|c|c|c|c|c|c|c|}
\hline & \multicolumn{3}{|c|}{ Patients with IIM } & \multicolumn{3}{|c|}{ General Population } & \multirow[b]{2}{*}{$\mathrm{RD}(95 \% \mathrm{CI})$} & \multirow[b]{2}{*}{ HR $(95 \% \mathrm{CI})$} \\
\hline & $\mathrm{ACS}, \mathrm{n}$ & $\mathrm{F} / \mathrm{U}, \mathrm{PY}$ & Rate $(95 \% \mathrm{CI})^{\dagger}$ & $\mathrm{ACS}, \mathrm{n}$ & $\mathrm{F} / \mathrm{U}, \mathrm{PY}$ & Rate $(95 \% \mathrm{CI})^{\dagger}$ & & \\
\hline $\mathrm{ACS}$ & 53 & 3402 & $15.6(11.7-20.4)$ & 313 & 41,783 & $7.5(6.7-8.4)$ & $5.7(2.2-9.3)$ & $2.4(1.8-3.2)$ \\
\hline UA & 2 & 3402 & $0.6(0.1-2.1)$ & 28 & 41,783 & $0.7(0.4-1.0)$ & $-0.2(-0.5$ to 0.1$)$ & $0.9(0.2-3.7)$ \\
\hline NSTEMI & 17 & 3402 & $5.0(2.9-8.0)$ & 103 & 41,783 & $2.5(2.0-3.0)$ & $1.8(-0.1$ to 3.8$)$ & $2.3(1.4-3.9)$ \\
\hline STEMI & 34 & 3402 & $10(6.9-14.0)$ & 182 & 41,783 & $4.4(3.7-5.0)$ & $4.0(1.2-6.8)$ & $2.8(1.9-4.0)$ \\
\hline \multicolumn{9}{|l|}{ Sex } \\
\hline Female & 30 & 1934 & $15.5(10.5-22.1)$ & 136 & 24,179 & $5.6(4.7-6.7)$ & $7.4(2.6-12.2)$ & $3.4(2.3-5.0)$ \\
\hline Male & 23 & 1469 & $15.7(9.9-23.5)$ & 177 & 17,604 & $10.1(8.6-11.7)$ & $3.9(-1.4$ to 9.2$)$ & $1.8(1.2-2.8)$ \\
\hline \multicolumn{9}{|c|}{ Age group, yrs } \\
\hline$<56$ & 7 & 1424 & $4.9(2.0-10.1)$ & 33 & 15,447 & $2.1(1.5-3.0)$ & $2.7(-0.9$ to 6.3$)$ & $2.4(1.1-5.5)$ \\
\hline $56-67$ & 11 & 1145 & $9.6(4.8-17.2)$ & 84 & 13,870 & $6.1(4.8-7.5)$ & $4.0(-1.8$ to 9.9$)$ & $1.6(0.8-3.0)$ \\
\hline $68-90$ & 35 & 834 & $42(29.2-58.4)$ & 196 & 12,466 & $15.7(13.6-18.1)$ & $26.5(12.5-40.6)$ & $2.8(1.9-4.1)$ \\
\hline \multicolumn{9}{|l|}{ Subset } \\
\hline DM & 8 & 1066 & $7.5(3.2-14.8)$ & 85 & 13,498 & $6.3(5.0-7.8)$ & $1.4(-4.0$ to 6.8$)$ & $1.2(0.6-2.5)$ \\
\hline Other IIM & 45 & 2337 & $19.3(14.0-25.8)$ & 228 & 28,276 & $8.1(7.1-9.2)$ & $11.4(5.7-17.1)$ & $2.5(1.8-3.4)$ \\
\hline
\end{tabular}

$\dagger$ Incidence rate per $1000 \mathrm{PY}$, CI estimated using a Poisson distribution. HR estimated by Cox models adjusted for age, sex, and county of residence at index year. Rate differences (RD) estimated using a Poisson model adjusted for age (tertiles) and sex. IIM: idiopathic inflammatory myopathy; F/U: followup; ACS: acute coronary syndrome; UA: unstable angina; STEMI: ST-elevation myocardial infarction; NSTEMI: non-STEMI; DM: dermatomyositis; PY: person-years.

Table 5. Number of ACS, followup, and HR for ACS stratified by time since diagnosis for patients with IIM and general population comparators.

\begin{tabular}{lccccr}
\hline \multirow{2}{*}{$\begin{array}{l}\text { Time since } \\
\text { Diagnosis, yrs }\end{array}$} & \multicolumn{2}{c}{ Patients with IIM } & \multicolumn{2}{c}{ General Population } & HR (95\% CI ${ }^{\dagger}$ \\
& ACS, n & Followup, PY & ACS, n & Followup, PY & \\
\hline$<1$ & 13 & 620 & 42 & 6759 & $3.6(1.9-6.7)$ \\
$1-5$ & 28 & 2385 & 158 & 28,408 & $2.4(1.6-3.7)$ \\
$5-12$ & 12 & 2481 & 113 & 32,445 & $1.8(1.0-3.3)$ \\
\hline
\end{tabular}

${ }^{\dagger}$ HR estimated by Cox models adjusted for age, sex, and county of residence at index year. IIM: idiopathic inflammatory myopathy; ACS: acute coronary syndrome; PY: person-years.

Canadian cohort study, a similar ACS incidence rate was found in patients with PM/DM (13.8/1000 PY), with an ACS relative risk of 1.95 (95\% CI 1.35-2.72) compared with the general population ${ }^{7}$. The risk of ACS in this study was highest in the first year after diagnosis and decreased in the subsequent years, similar to our results. We can hypothesize that some factors in the period following diagnosis such as introduction of immunosuppression or higher IIM disease activity might influence ACS risk. Further studies are necessary, however, to elucidate why this period is critical in the development of ACS in IIM. In our study, after IIM subset stratification, a 2-fold ACS risk increase was seen only in patients with IIM who were not affected by DM. These results differ from a previous large population-based study reporting HR for myocardial infarction in PM of 3.89 (95\% CI 2.28-6.65) and in DM of 2.92 (95\% CI 1.48-5.78) ${ }^{9}$. The discrepancy between our results could not be explained simply by differences in case and outcome definitions but could be due to the small number of events $(n=8)$ recorded in this subgroup.

In our study, a higher proportion of IIM individuals were receiving medication to treat $\mathrm{CV}$ diseases at baseline compared to the general population. However, IIM individuals were less exposed to statins and/or fibrates. Myalgias and mild elevation of serum creatinine kinase are frequent side effects of statins ${ }^{28}$. In certain IIM individuals at the early stage of disease, statins might be discontinued for suspicion of statin-induced muscle toxicity and might not be reintroduced afterward. This might affect the ACS risk in the IIM population. Even if medication data were available for only $60 \%$ of our cases/controls and could not be used in our model, we consider this sample representative and comparable to the rest of the cohort.

When comparing our IIM cohort with the general population, a 3 -fold increase in ACS relative risk was observed in elderly and female IIM individuals. To our knowledge, this is the first study to report a sex difference in the risk of developing ACS in patients with IIM when compared with the general population. Even if the crude incidences were similar between males and females, the relative risk of ACS when compared with the general population was clearly higher in females. In a Swedish

Personal non-commercial use only. The Journal of Rheumatology Copyright @ 2019 . All rights reserved. 
population-based RA study, such a sex difference in ACS risk was not found ${ }^{29}$. Despite being exposed to a similar inflammatory state, female patients with RA in the aforementioned study had a lower incidence rate of ACS compared to males. In our study, both sexes have similar incidence rates, suggesting that the mechanisms underlying ACS in the IIM population could differ from other rheumatologic diseases. This notion should also be taken into consideration when clinicians consider cardiac risk stratification in that population. Histopathologic data revealed that $40 \%$ of IIM individuals have myocarditis on autopsy, a condition that might be clinically difficult to differentiate from an ACS without advanced imaging such as cardiac magnetic resonance ${ }^{30}$. In addition, a previous cross-sectional study of 47 patients with $\mathrm{PM} / \mathrm{DM}$ described myocardial inflammation in $60 \%$ of them ${ }^{31}$. Even if those individuals were not all considered to have myocarditis, myocardial inflammation may surely influence ACS diagnosis in that population. The distinction between those 2 conditions is important, however, because myocarditis requires aggressive immunosuppression.

This is one of the largest population-based studies to assess ACS risk in an incident IIM cohort that included patients with IBM. The inclusion of this particular subset might underestimate the ACS incidence rate in the "other IIM" subgroup. Evidence suggests that cardiac abnormalities are not increased in IBM compared to elderly individuals ${ }^{32}$. To our knowledge, cardiac involvement in IBM at disease onset has never been assessed, probably owing to the usually long delay before diagnosis. This underlines a limitation of our study, in which coding overlap limited our ability to divide our IIM cases into the different classic IIM subgroups (PM, DM, IBM). The large "other IIM" subgroup is therefore heterogeneous, and most likely contains subsets that are more at risk of ACS than others. Even if we based our case ascertainment on a robust case definition for IIM, there is still a risk of misclassification given that our IIM cases were not confirmed by imaging or histopathology. Additionally, diagnosis is often delayed in IIM, with patients developing symptoms several years before diagnosis. Our approach was to exclude those subjects who might have had their ACS in their "latency period," which would underestimate the ACS risk in our cohort. It is possible that some comorbidities such as hypertension and atrial fibrillation were detected more frequently in patients with IIM as a result of a surveillance bias. Administrative data also did not allow for stratification by autoantibody status. Phenotypes based on autoantibody profiles are characterized by different extramuscular involvement and might be associated with different ACS risk. Smoking and obesity are known risk factors for CV disease and are important confounders in this type of study, but were not available for inclusion in our analyses.

This large population-based study clearly demonstrates an increased risk of ACS in IIM, particularly in the year following diagnosis and in older individuals. Future colla- borative studies are needed to understand the mechanisms underlying ACS in IIM, taking in consideration IIM subsets and autoantibody profiles.

\section{ONLINE SUPPLEMENT}

Supplementary material accompanies the online version of this article.

\section{REFERENCES}

1. Lundberg IE, Tjärnlund A, Bottai M, Werth VP, Pilkington C, Visser M, et al; International Myositis Classification Criteria Project consortium, The Euromyositis register and The Juvenile Dermatomyositis Cohort Biomarker Study and Repository (JDRG) (UK and Ireland). 2017 European League Against Rheumatism/American College of Rheumatology classification criteria for adult and juvenile idiopathic inflammatory myopathies and their major subgroups. Ann Rheum Dis 2017;76:1955-64.

2. Marie I. Morbidity and mortality in adult polymyositis and dermatomyositis. Curr Rheumatol Rep 2012;14:275-85.

3. Dobloug GC, Svensson J, Lundberg IE, Holmqvist M. Mortality in idiopathic inflammatory myopathy: results from a Swedish nationwide population-based cohort study. Ann Rheum Dis 2018;77:40-7.

4. Lai YT, Dai YS, Yen MF, Chen LS, Chen HH, Cooper RG, et al. Dermatomyositis is associated with an increased risk of cardiovascular and cerebrovascular events: a Taiwanese population-based longitudinal follow-up study. Br J Dermatol 2013;168:1054-9.

5. Linos E, Fiorentino D, Lingala B, Krishnan E, Chung L. Atherosclerotic cardiovascular disease and dermatomyositis: an analysis of the Nationwide Inpatient Sample survey. Arthritis Res Ther 2013;15:R7.

6. Zöller B, Li X, Sundquist J, Sundquist K. Risk of subsequent coronary heart disease in patients hospitalized for immune-mediated diseases: a nationwide follow-up study from Sweden. PLoS One 2012;7:e33442.

7. Tisseverasinghe A, Bernatsky S, Pineau CA. Arterial events in persons with dermatomyositis and polymyositis. J Rheumatol 2009;36:1943-6.

8. Ungprasert P, Suksaranjit P, Spanuchart I, Leeaphorn N, Permpalung N. Risk of coronary artery disease in patients with idiopathic inflammatory myopathies: a systematic review and meta-analysis of observational studies. Semin Arthritis Rheum 2014;44:63-7.

9. Rai SK, Choi HK, Sayre EC, Aviña-Zubieta JA. Risk of myocardial infarction and ischaemic stroke in adults with polymyositis and dermatomyositis: a general population-based study. Rheumatology 2016;55:461-9.

10. Avina-Zubieta JA, Thomas J, Sadatsafavi M, Lehman AJ, Lacaille D. Risk of incident cardiovascular events in patients with rheumatoid arthritis: a meta-analysis of observational studies. Ann Rheum Dis 2012;71:1524-9.

11. Yurkovich M, Vostretsova K, Chen W, Aviña-Zubieta JA. Overall and cause-specific mortality in patients with systemic lupus erythematosus: a meta-analysis of observational studies. Arthritis Care Res 2014;66:608-16.

12. Limaye V, Lester S, Blumbergs P, Roberts-Thomson PJ. Idiopathic inflammatory myositis is associated with a high incidence of hypertension and diabetes mellitus. Int J Rheum Dis 2010;13:132-7.

13. Schwartz T, Diederichsen LP, Lundberg IE, Sjaastad I, Sanner H. Cardiac involvement in adult and juvenile idiopathic inflammatory myopathies. RMD Open 2016;2:e000291.

14. Libby P. Inflammation in atherosclerosis. Arterioscler Thromb Vasc Biol 2012;32:2045-51.

15. Haupt HM, Hutchins GM. The heart and cardiac conduction system

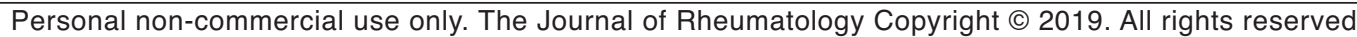


in polymyositis-dermatomyositis: a clinicopathologic study of 16 autopsied patients. Am J Cardiol 1982;50:998-1006.

16. Helmers SB, Jiang X, Pettersson D, Wikman AL, Axelman P, Lundberg $\AA$, et al. Inflammatory lung disease a potential risk factor for onset of idiopathic inflammatory myopathies: results from a pilot study. RMD Open 2016;2:e000342.

17. Bernatsky S, Linehan T, Hanly JG. The accuracy of administrative data diagnoses of systemic autoimmune rheumatic diseases. J Rheumatol 2011;38:1612-6.

18. McCormick N, Lacaille D, Bhole V, Avina-Zubieta JA. Validity of myocardial infarction diagnoses in administrative databases: a systematic review. PLoS One 2014;9:e92286.

19. Ljung L, Simard JF, Jacobsson L, Rantapää-Dahlqvist S, Askling J; Anti-Rheumatic Therapy in Sweden (ARTIS) Study Group. Treatment with tumor necrosis factor inhibitors and the risk of acute coronary syndromes in early rheumatoid arthritis. Arthritis Rheum 2012;64:42-52.

20. Holmqvist M, Gränsmark E, Mantel A, Alfredsson L, Jacobsson LT, Wallberg-Jonsson S, et al. Occurrence and relative risk of stroke in incident and prevalent contemporary rheumatoid arthritis. Ann Rheum Dis 2013;72:541-6.

21. Go AS, Hylek EM, Borowsky LH, Phillips KA, Selby JV, Singer DE. Warfarin use among ambulatory patients with nonvalvular atrial fibrillation: the anticoagulation and risk factors in atrial fibrillation (ATRIA) study. Ann Intern Med 1999;131:927-34.

22. McCormick N, Lacaille D, Bhole V, Avina-Zubieta JA. Validity of heart failure diagnoses in administrative databases: a systematic review and meta-analysis. PLoS One 2014;9:e104519.

23. Khokhar B, Jette N, Metcalfe A, Cunningham CT, Quan H, Kaplan $\mathrm{GG}$, et al. Systematic review of validated case definitions for diabetes in ICD-9-coded and ICD-10-coded data in adult populations. BMJ Open 2016;6:e009952.

24. Henderson T, Shepheard J, Sundararajan V. Quality of diagnosis and procedure coding in ICD-10 administrative data. Med Care 2006;44:1011-9.

25. Cutler DM, Lleras-Muney A. Understanding differences in health behaviors by education. J Health Econ 2010;29:1-28.

26. Fay MP, Feuer EJ. Confidence intervals for directly standardized rates: a method based on the gamma distribution. Stat Med 1997;16:791-801.

27. Fine JP, Gray RJ. A proportional hazards model for the subdistribution of a competing risk. J American Stat Assoc 1999;94:496-509.

28. Parker BA, Capizzi JA, Grimaldi AS, Clarkson PM, Cole SM, Keadle J, et al. Effect of statins on skeletal muscle function. Circulation 2013;127:96-103.

29. Holmqvist M, Ljung L, Askling J. Acute coronary syndrome in new-onset rheumatoid arthritis: a population-based nationwide cohort study of time trends in risks and excess risks. Ann Rheum Dis 2017;76:1642-7.

30. Gupta R, Wayangankar SA, Targoff IN, Hennebry TA. Clinical cardiac involvement in idiopathic inflammatory myopathies: a systematic review. Int J Cardiol 2011;148:261-70.

31. Rosenbohm A, Buckert D, Gerischer N, Walcher T, Kassubek J, Rottbauer W, et al. Early diagnosis of cardiac involvement in idiopathic inflammatory myopathy by cardiac magnetic resonance tomography. J Neurol 2015;262:949-56.

32. Cox FM, Delgado V, Verschuuren JJ, Ballieux BE, Bax JJ, Wintzen AR, et al. The heart in sporadic inclusion body myositis: a study in 51 patients. J Neurol 2010;257:447-51. 